Case Report

\title{
A Case of Pediatric Cerebral Hemorrhage Complicated with Infective Endocarditis
}

\author{
Keishiro Furuie, Hiroshi Tamura*, Osamu Matsuo, Hiroshi Mitsubuchi \\ Department of Pediatrics, Faculty of Life Sciences, Kumamoto University, Kumamoto, Japan \\ Email address: \\ bohm1905HT@kuh.kumamoto-u.ac.jp (H. Tamura) \\ ${ }^{*}$ Corresponding author
}

\section{To cite this article:}

Keishiro Furuie, Hiroshi Tamura, Osamu Matsuo, Hiroshi Mitsubuchi. A Case of Pediatric Cerebral Hemorrhage Complicated with Infective Endocarditis. American Journal of Pediatrics. Vol. 5, No. 4, 2019, pp. 267-269. doi: 10.11648/j.ajp.20190504.27

Received: October 6, 2019; Accepted: November 4, 2019; Published: November 25, 2019

\begin{abstract}
The mortality rate associated with cerebral hemorrhage as a complication of IE is extremely high despite the development of preventive and antibiotic therapy. We report a 13-year-old female whose previous medical history includes Jeune syndrome and mitral regurgitation. She was taken to a nearby hospital because of high body temperature. She had no symptoms of cold or gastrointestinal disturbance and was diagnosed with upper respiratory tract inflammation. On the subsequent day, she experienced a seizure and was brought to the emergency department of the general hospital. Her head computed tomography (CT) revealed bleeding and mild cerebral edema in the left occipital lobe. A mild systolic murmur was audible upon auscultation. She presented with carious tooth and Janeway lesions. Methicillin-resistant Staphylococcus aureus (MRSA) was positive on blood culture examination, cerebrospinal fluid examination was negative. A diagnosis of IE was established based on the Duke criteria. Antibiotic therapy was administered. Thereafter, the symptoms disappeared without any adverse effects, and antibiotic administration was ended in six weeks. After the discharge, dental treatment was initiated. There was no recurrence of IE at 3 years after the onset, and no sequelae were observed. Although the prevalence of cerebral hemorrhage as a complication of IE is low, the associated mortality rate is extremely high despite the development of preventive and antibiotic therapy. Moreover, there is no consensus regarding the treatment method. In this case, only a conservative treatment was performed without sequelae. Pediatric patients with stroke associated with IE may have better outcome than adults. It is important to suspect IE and to provide immediate or early treatment if fever and central nervous symptoms are observed in cases with underlying heart disease.
\end{abstract}

Keywords: Cerebral Hemorrhage, Infective Endocarditis, Children

\section{Introduction}

Infective endocarditis (IE) is a systemic disease that exhibits various symptoms and is associated with a high frequency of neurological complications. Among them, stroke is a typical neurological complication that is present in $12 \%$ $25 \%$ of all IE cases [1]. Neurological complications of IE are considered major risk factors for increased morbidity and mortality [2]. Neurological complications may affect both the medical therapy and the optimal timing for surgery [3]. Here, we report a case of IE discovered based on intracerebral hemorrhage and treated without sequelae.

\section{Case}

A 13-year-old girl with high body temperature was taken to a nearby hospital. She had no symptoms of cold or gastrointestinal disturbance and was diagnosed with upper respiratory tract inflammation. On the subsequent day, she experienced a seizure and was brought to the emergency department of the general hospital. Her consciousness level was low, and her initial Glasgow Coma Scale score was 6 (E1V1M4). Her head computed tomography (CT) revealed bleeding and mild cerebral edema in the left occipital lobe (Figure 1). She was transferred to our hospital for further 
examination and treatment. She had a history of Jeune syndrome and mitral valve regurgitation. Her body temperature was $38.4^{\circ} \mathrm{C}$, heart rate $154 \mathrm{bpm}$, respiration rate $50 \mathrm{bpm}$, and blood pressure $127 / 78 \mathrm{mmHg}$. A mild systolic murmur was audible upon auscultation. She presented with carious tooth and Janeway lesions. On laboratory examination, her white blood cell count was $19,000 / \mu \mathrm{L}$, hemoglobin concentration $14.1 \mathrm{~g} / \mathrm{dL}$, and C-reactive protein level $17.43 \mathrm{mg} / \mathrm{dL}$. Methicillin-resistant Staphylococcus aureus (MRSA) was positive on blood culture examination, cerebrospinal fluid examination was negative (performed previously at another hospital), and MRSA findings were positive in the blood culture at our hospital. Transthoracic echocardiography revealed a fractional shortening of $30.9 \%$ and a vegetation measuring $7 \times 9 \mathrm{~mm}$ on the anterior cusp of the mitral valve, and the two cusps of the mitral valve demonstrated mild mitral regurgitation. A diagnosis of IE was established based on the Duke criteria. Antibiotic therapy with $350 \mathrm{mg}$ of vancomycin was administered every $4 \mathrm{~h}$, initiated as per the Guidelines for the Prevention and Treatment of Infective Endocarditis released by the Japanese Circulation Society in 2008. Renal toxicity due to vancomycin appeared after use; therefore, the medication was changed to linezolid. Thereafter, the symptoms disappeared without any adverse effects, and antibiotic administration was ended in six weeks. Moreover, echocardiography did not show deterioration on the weekly follow-ups. Blood culture test was performed thrice and confirmed negativity. Bleeding from a bacterial cerebral aneurysm was suspected on the basis of the clinical course; therefore, head CT scanning and magnetic resonance imaging angiography were performed. However, no aneurysm, infarction, or vascular malformation was observed. Furthermore, brain angiography did not show aneurysm. After the discharge, dental treatment was initiated. There was no recurrence of IE at 3 years after the onset, and no sequelae were observed.

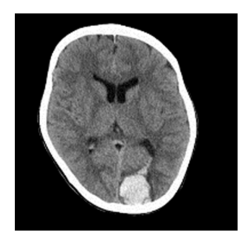

Figure 1. Computed tomography showing massive bleeding and mild cerebral edema in the left occipital lobe.

\section{Discussion}

Childhood infectious endocarditis is still found at a constant frequency and with high mortality rate even if the prevention and antibiotic therapy are developed.

The etiology of IE is known in about $25 \%$ of cases. Approximately half of them are due to dental treatments, cardiac surgery, and cardiac catheterization. In addition, obstetric procedures, urinary catheters, hemodialysis, and drugs (steroids and antibiotics) may also trigger it [4]. In this case, it was thought that mitral valve insufficiency was followed once every six months, but carious teeth were untreated, which induced infective endocarditis.

Fever is usually the presenting symptom upon admission in almost all cases, whereas heart murmur is the cause in about $80 \%$ [5]. General malaise, muscle pain, joint pain, headache, nausea, and vomiting are common symptoms. Osler nodules, Janeway lesion, and petechial hemorrhage, which are the classical skin symptoms, are found in advanced cases not in those with early diagnosis and treatment [6]. In this case, fever and seizure were first observed. About $30 \mathrm{~h}$ after the onset of fever, cerebral hemorrhage occurred and Janeway lesion was found, which were thought to progress relatively faster.

In complications of IE, congestive heart failure is the most common and shows at $50 \%$ of the cases. Other complications include the renal, pulmonary, and cranial nerve. The cranial nerve complication has the highest fatality and is reported to occur in $20 \%$ to $40 \%$ of cases of IE, such as meningoencephalitis, cerebral abscess, cerebral hemorrhage, cerebral embolism, and cerebral aneurysm [6]. Although its fatality rate tends to be lower due to the development of antibiotics, it is still one of the major death causes of IE. Cerebral hemorrhage in IE is presumed to result from three mechanisms of hemorrhagic infarction, rupture of infectious aneurysm, and vasculitis at embolic site [7]. In this case, the cerebrovascular breakdown was thought to be caused by vasculitis at the embolic site not by hemorrhagic aneurysm, which was examined immediately after its onset.

A case of cerebral hemorrhage associated with IE reported in children was investigated in literature (Table 1). There were 13 case reports, including this case, which were examined. The age ranged from 1 month to 17 years (median 11 years old), and there were 9 females and 4 males.

Table 1. Case of pediatric cerebral hemorrhage complicated with infective endocarditis reported in the literature.

\begin{tabular}{|c|c|c|c|c|c|c|c|c|}
\hline Case & $\begin{array}{l}\text { Age } \\
\text { (years) }\end{array}$ & Sex & Organism & $\begin{array}{l}\text { Cardiac history/ } \\
\text { location of Vegetation }\end{array}$ & $\begin{array}{l}\text { Blood vessel } \\
\text { involved }\end{array}$ & $\begin{array}{l}\text { Mycotic } \\
\text { Aneurysm }\end{array}$ & Outcome & Reference \\
\hline 1 & 3 weeks & female & Staphylococcus aureus & $-/ \mathrm{MV}$ & Multiple & Yes & $\begin{array}{l}\text { Dysphagia, axial } \\
\text { hypotonia }\end{array}$ & 8 \\
\hline 2 & 1 & female & - & VSD/TV & Right MCA & NR & Dead & 9 \\
\hline 3 & 1 & Female & candida glabrata & VSD/MV & Both ICA & NR & Dead & 9 \\
\hline 4 & 3 & female & Streptococcus sanguinis & $\mathrm{MR} / \mathrm{NR}$ & right $\mathrm{MCA}$ & Yes & $\begin{array}{l}\text { Left facial } \\
\text { paralysis }\end{array}$ & 6 \\
\hline 5 & 9 & male & Granulicatella elegans & Coactation/AV & right $\mathrm{MCA}$ & Yes & Intact & 8 \\
\hline 6 & 9 & male & $\begin{array}{l}\text { Staphylococcus } \\
\text { epidermidis }\end{array}$ & Coactation/NR & right $\mathrm{PCA}$ & Yes & Intact & 10 \\
\hline 7 & 11 & female & Alph- Streptococcus & redundant MV/MV & right $\mathrm{MCA}$ & No & NR & 8 \\
\hline 8 & 13 & female & MRSA & $\mathrm{MR} / \mathrm{MV}$ & Left PCA & No & Intact & This case \\
\hline 9 & 14 & female & Streptococcus sanguinis & VSD/MV & right $\mathrm{PCA}$ & Yes & Left homonymous & 6 \\
\hline
\end{tabular}




\begin{tabular}{lllllllll}
\hline Case & $\begin{array}{l}\text { Age } \\
\text { (years) }\end{array}$ & Sex & Organism & $\begin{array}{l}\text { Cardiac history/ } \\
\text { location of Vegetation }\end{array}$ & $\begin{array}{l}\text { Blood vessel } \\
\text { involved }\end{array}$ & $\begin{array}{l}\text { Mycotic } \\
\text { Aneurysm }\end{array}$ & Outcome & Reference \\
\hline & & & & & & & hemianopia \\
10 & 15 & male & Staphylococcus aureus & TGA/AV & left MCA & No & Intact & 8 \\
11 & 16 & female & Group B Streptococcus & -/MV & left MCA & Yes & Right hemiplegia & 8 \\
12 & 16 & male & - & left MCA & NR & Intact & 9 \\
13 & 17 & female & Abiotrophia defectiva & NR/MV & right MCA & Yes & spastic tetraparesis & 11 \\
\hline
\end{tabular}

Abbreviation: NR; not reported, VSD; ventricular septal defect, MR; mitral regurgitation, TGA; transposition of the great arteries, MV; mitral valve, TV; tricuspid valve, AV; aortic valve, MCA; middle cerebral artery, ICA; internal cerebral artery, PCA; posterior cerebral artery S, aureus was reported to be associated with an increased risk of stroke [12]. In this study, out of 13 cases of IE, S, aureus were identified in 3 patients. Due to this lack of data, S. aureus infection's impact on the risk of stroke in children could not be further analyzed.

Out of 12 cases of IE, 9 had a history of heart disease; out of 11 cases with vegetation, 7 involved the mitral valve and 3 the aortic valve. The incidence of stroke was significantly higher in patients with left-sided endocarditis than those with right-sided endocarditis [9]. These results imply that stroke was more common in children with left-sided endocarditis.

The bleeding site was the middle cerebral artery in 9 cases, and cerebral aneurysm was found in 7 cases. Infectious cerebral aneurysms are different from normal cerebral aneurysms and are often found in the middle cerebral artery [12].

The mortality rate of the patients with stroke was significantly higher than that of those without. Previous studies of adult patients with endocarditis have found a higher mortality rate in those with neurological complications [11]. In the study, 2 patients were one-year-old, 5 patients had sequelae, and 5 patients were intact. Previous studies of adult patients with endocarditis have found a higher mortality rate in those with neurological complications [13]. These findings suggest that pediatric patients with stroke associated with IE may have better outcome than adults.

\section{Conclusion}

Although the prevalence of cerebral hemorrhage as a complication of IE is low, the associated mortality rate is extremely high despite the development of preventive and antibiotic therapy. Moreover, there is no consensus regarding the treatment method.

In this case, only a conservative treatment was performed without sequelae, which corroborates to the finding that pediatric patients with stroke associated with IE may have better outcome than adults.

Therefore, it is important to suspect IE and to provide immediate or early treatment if fever and central nervous symptoms are observed in cases with underlying heart disease.

\section{Conflict of Interest}

The authors no conflict of interest.

\section{References}

[1] Thuny F, Avierinos JF, Tribouilloy, et al. Impact of cerebrovascular complications on mortality and neurologic outcome during infective endocarditis: a prospective multicentre study. Eur Heart J. 2007; 28: 1155-1161.
[2] Murdoch DR, Corey GR, Hoen B, et al. Clinical presentation, etiology, and outcome of infective endocarditis in the $21^{\text {st }}$ century: the International Collaboration on Endocarditis-Prospective Cohort Study. Arch Intern Med. 2009; 169: 463-473.

[3] Duval X, Delahaye F, Tattevin P, et al. Temporal trends in infective endocarditis in the context of prophylaxis guideline modifications: three successive population-based surveys. J Am Coll Cardiol. 2012; 59: 1968-1976.

[4] Lawrence L, Pelletier Jr, and Petersdorg R. Infective Endocarditis: A review of 125 cases from the University of Washington Hospitals. Medicine. 1977; 56: 1963-1972.

[5] Duranate-Mangoni E, Bradley S, Selton-Suty C, et al. Current features of infective endocarditis in elderly patients: results of the International Collaboration on Endocarditis Prospective Cohort Study. Arch Intern Med 2008; 168: 2095-2103.

[6] Keiko M, Katsumi A, Kei $\mathrm{N}$ et al, The two cases of infective endocarditis complicated rupture of bacterial aneurysm. Heart, 1986; 18: 210-217.

[7] Okazaki S, Yoshioka D, Sakaguchi m, et al. Acute ischemic brain lesions in infective endocarditis: incidence, related factors, and postoperative outocome. Cerebrovase Dis. 2013; 35: $155-162$.

[8] Charu V, Mark S. P. Pediatric Endocarditis and Stroku: A single Center Retrospective Review of Seven Cases. Pediatr Neurol. 2008; 38: 243-247.

[9] Gui-fang Cao, Qi, Pediatric and stroke: A 13-year single center review. Pediatr Neurol. 2019; 90: 56-60.

[10] Christopher E, Dimitrios N, Ali S, et al, HydroCoil embolization of a ruptured aneurysm in a pediatric patient: case report and review of the literature. Childs Nerv Syst. 2007; 23: 707-712.

[11] Filipa F L, Lurdes L, Antonio S, et al, Myotic brain aneurysm and cerebral hemorrhagic stroke: a pediatric case report. Eur J pediatr, 2013; 172: 1285-1286.

[12] Salagado AV, Furlan AJ, Keys TF, et al. Neurologic complications of endocarditis: a 12-year single-center experience. Neurology. 1989; 39: 173-178.

[13] Chen $\mathrm{CH}$, Lo MC, Hwang KL, et al. Infective endocarditis with neurologic complications: 10-year experience. J Microbiol Immunol Infect. 2001; 34: 119-124. 